\title{
NUMERICAL APPROXIMATION OF NEMATIC LIQUID CRYSTAL FLOWS GOVERNED BY THE ERICKSEN-LESLIE EQUATIONS*
}

\author{
NoEL J. WALKINGTON ${ }^{1}$
}

\begin{abstract}
Numerical approximation of the flow of liquid crystals governed by the Ericksen-Leslie equations is considered. Care is taken to develop numerical schemes which inherit the Hamiltonian structure of these equations and associated stability properties. For a large class of material parameters compactness of the discrete solutions is established which guarantees convergence.
\end{abstract}

Mathematics Subject Classification. 76A15, 65M12, 65M60, 76M10.

Received January 5, 2010. Revised June 14, 2010.

Published online November 30, 2010.

\section{INTRODUCTION}

Numerical approximation of the Ericksen-Leslie equations is considered. These equations model the motion of nematic liquid crystalline fluids and take the form

$$
\begin{aligned}
\rho \dot{v}-\operatorname{div}(T) & =\rho f, & \operatorname{div}(v) & =0, \\
\left(\rho \bar{r}^{2}\right) \ddot{d}+g-\operatorname{div}(C) & =\rho m, & |d| & =1,
\end{aligned}
$$

along with appropriate initial and boundary conditions. Here $v$ is the fluid velocity, $d$ is a unit vector characterizing the orientation of the liquid crystal molecules, and $\dot{d}=d_{t}+(v \cdot \nabla) d$ is the convected time derivative. The first equation models the balance or linear momentum and the second the balance of angular momentum. The quantity $\bar{r}$ appearing in the inertial term of the angular momentum equation represents the radius of gyration of the molecules and is small (order the length of the molecule). For this reason this term is usually neglected; it is only included here to illustrate the origin of the equation for $d$, and to emphasize that the equation changes type from hyperbolic to parabolic when this term is omitted.

The Cauchy stress of a nematic liquid crystal takes the form

$$
T=-p I+T_{e}+T_{v} \equiv-p I-(\nabla d)^{T}\left[\frac{\partial \mathcal{W}}{\partial \nabla d}\right]+T_{v}
$$

Keywords and phrases. Liquid crystal, Ericksen-Leslie equations, numerical approximation.

* Supported in part by National Science Foundation Grants DMS-0811029. This work was also supported by the NSF through the Center for Nonlinear Analysis.

1 Department of Mathematics, Carnegie Mellon University, Pittsburgh, PA 15213, USA. noelw@andrew.cmu.edu 
where $T_{e}$ is the elastic stress, and the viscous stress is

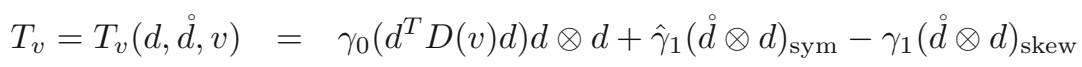

$$
\begin{aligned}
& +\mu D(v)+\hat{\gamma}_{2}(D(v) d \otimes d)_{\mathrm{sym}}-\gamma_{2}(D(v) d \otimes d)_{\text {skew }} .
\end{aligned}
$$

Here $D(v)$ and $W(v)$ are the skew and symmetric parts of $\nabla v ; \stackrel{d}{d}=\dot{d}-W(v) d$; and $\mathcal{W}=\mathcal{W}(d, \nabla d)$ is the elastic energy of the nematic [11]. The stress couples in the angular momentum equation are

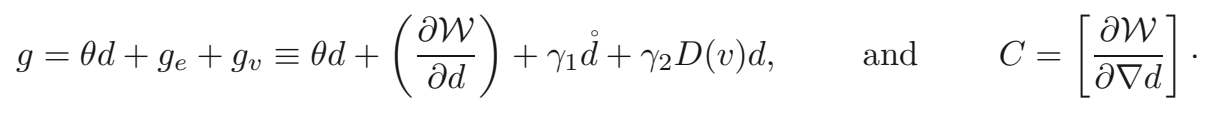

We have chosen to group the viscous terms into symmetric and skew terms ${ }^{2}$. In terms of the usual constants

$$
\gamma_{0}=\alpha_{1}, \gamma_{1}=\alpha_{3}-\alpha_{2}, \hat{\gamma}_{1}=\alpha_{3}+\alpha_{2}, \mu=\alpha_{4}, \gamma_{2}=\alpha_{6}-\alpha_{5}, \hat{\gamma}_{2}=\alpha_{6}+\alpha_{5} .
$$

Restrictions on these constants to guarantee that the viscous terms are dissipative are given in Section 2.2. In particular $\gamma_{1}$ is required to be non-negative and below it will be assumed that $\gamma_{1}>0$ so that the angular momentum equation becomes parabolic upon setting $\bar{r}=0$.

To date there is no satisfactory existence theory for the Ericksen-Leslie equations in their full generality. The non-convex constraint on the director presents many challenges to the existence theory and to the construction of numerical schemes. Frequently a penalized approximation of this constraint is used to circumvent these difficulties. The other major difficulty arises from the quartic terms in the Oseen Frank energy $\mathcal{W}$. The compactness properties of the corresponding Euler Lagrange equation, which is vector valued, are not well developed [30]. For this reason we will consider the penalized Ericksen-Leslie equations and quadratic elastic energies, in which case the elastic terms are linear in $d$.

In this paper we establish convergence of numerical schemes for the penalized approximation of the EricksenLeslie equations with a quadratic elastic energy. The Hamiltonian structure facilitates the development of conforming $C^{0}$ schemes which have solutions bounded independently of the penalty parameter. The ideas introduced here can also be used to simplify the Hermite and mixed methods commonly used for the simplified model equations introduced by Lin and Liu [25]. Our analysis allows spatial discretization by finite elements (or spectral bases) of arbitrary order; however, for technical reasons the second order Crank Nicolson time stepping scheme (or the first order implicit Euler scheme) is considered; the extension to higher order time stepping schemes is not immediate.

In the mechanics literature physically correct expressions for the elastic energy and dissipative terms are developed under the assumption that the constraints $\operatorname{div}(v)=0$ and $|d|=1$ are satisfied exactly. Numerical approximations only approximate these constraints and it is important to extend the definitions of the energy and dissipative terms so that the discrete schemes retain the essential properties of the underlying equations. This topic is taken up in Section 2 where the Hamiltonian structure, energy estimate, and weak statements, of the Ericksen-Leslie equations are elucidated. Section 3 then considers the numerical approximation of solutions to the weak problem and establishes convergence.

\subsection{Related results}

Variational principles to characterize the equilibrium states of (nematic) liquid crystals were developed by Oseen [31] and Frank [11]. Equations modeling the dynamics of a liquid crystal flow were developed by Ericksen [8,10] and Leslie [20,21] within the context of continuum mechanics. The texts by Virga [33] and Stewart [32] provide a modern derivation of these models.

Hardt, Kinderlehrer and Lin [15-17] analyze the variational problem characterizing equilibrium configurations. The constraint $|d|=1$ on the director gives rise to many of the phenomena encountered with the harmonic

\footnotetext{
${ }^{2}$ Sometimes $g$ is defined to have the opposite sign.
} 
mapping problem [4,19,22,24]. In [23] Lin introduced the simplified system consisting of a Navier-Stokes type of equation coupled with a gradient flow equation (see Sect. 2.5 below). This system retains some of the essential properties of the original Ericksen-Leslie equations and an analysis of this problem (with the constraint penalized) was carried out in $[25,26]$.

The analysis by Lin and Liu in [25] facilitated the development and analysis of numerical schemes for this class of problems. Direct application of their ideas results in numerical schemes which require either Hermite elements or a mixed method [28,29]. Subsequently there have been many developments and refinements. Various mixed methods have been considered in [2,3,13]; spectral schemes have been developed in [6]; methods with estimates independent of the penalty parameter were developed in [3,27]; and "semi-implicit" schemes which only require the solution of a linear system at each time step are developed in [13]. All of these schemes for this simplified model utilize the first order implicit Euler stepping scheme to evolve the solution in time. In [34] the author developed numerical schemes of arbitrary order in space and time for this class of problems and established convergence.

\subsection{Notation}

It will be assumed that the equations are to be solved in a bounded connected Lipschitz domain $\Omega \subset \mathbb{R}^{n}$ with $n=2$ or $n=3$. Occasionally formulae or estimates for the two and three dimensional case differ slightly. In this situation the more technical three dimensional case will be considered. Inner products of vectors in $\mathbb{R}^{n}$ will be denoted by $(.,$.$) as will be the Frobenius inner product of matrices in \mathbb{R}^{n \times n}$; the corresponding norms are written as $|$.$| . If a \in \mathbb{R}^{n}$ then $\operatorname{rot}(a)_{i k}=\epsilon_{i j k} a_{j}$ denotes the skew matrix with axial vector $a \in \mathbb{R}^{n}$; that is, $\operatorname{rot}(a) b=a \times b$.

Divergences of vectors $v \in \mathbb{R}^{n}$ and matrices $T \in \mathbb{R}^{n \times n}$ are denoted $\operatorname{div}(v)=v_{i, i}$ and $\operatorname{div}(T)_{j}=T_{i j, j}$ respectively. Here indices after the comma represent partial derivatives and the summation convention is used. Gradients of vector valued quantities are interpreted as matrices, $(\nabla v)_{i j}=v_{i, j}$. The symmetric part of the such a gradient is written as $D(u)$, and the skew part as $W(v)$.

Standard notation is used for the Lebesgue spaces, $L^{p}(\Omega)$, and Sobolev spaces, $W^{1, p}(\Omega), W_{0}^{1, p}(\Omega)$, and $H^{1}(\Omega)=W^{1,2}(\Omega)$. Spaces of Bochner integrable functions from a time interval $[0, T]$ to a Banach space $U$ will be denoted as $L^{p}[0, T ; U]$, and $\mathcal{P}_{\ell}[0, T ; U]$ will indicate functions of the form $u(t)=\sum_{i=0}^{\ell} p_{i}(t) u_{i}$ with $u_{i} \in U$ and $p_{i} \in \mathcal{P}_{\ell}(0, T)$, the space of polynomials with degree less than or equal to $\ell$. The derivative of $u \in L^{p}[0, T ; U]$ is denoted as $u^{\prime}$ or $u_{t}$. The notation $U \hookrightarrow H$ denotes a continuous embedding of Banach spaces, and $U \hookrightarrow H$ denotes a compact embedding.

\section{Structure of the ERICKSEn-Leslie EQUATions}

In this section the essential mathematical properties of the Ericksen-Leslie equations are reviewed. These equations have a natural Hamiltonian structure which facilitates the development of numerical schemes which inherit the natural energy estimate.

\subsection{Hamiltonian structure and energy estimate}

Equations (1.1)-(1.2) may be derived from Hamilton's principle. If $X \in \Omega_{r}$ denotes a Lagrangian reference domain, and $x(X, t)$ denotes the flow map, then

$$
\begin{aligned}
\delta \int_{t_{1}}^{t_{2}} \int_{\Omega_{r}}\left\{-\left(\rho_{r} / 2\right)\left(|\dot{x}|^{2}+\bar{r}^{2}|\dot{d}|^{2}\right)+\mathcal{W}\right\} \mathrm{d} X \mathrm{~d} t=\int_{t_{1}}^{t_{2}} \int_{\Omega(t)}\{(f, \delta x)+(m, \delta d) & -\left(T_{v}, \nabla \delta x\right) \\
& \left.+\left(T_{v}, \operatorname{rot}(\delta d)\right)-\left(C_{v}, \nabla \delta d\right)\right\} \mathrm{d} x \mathrm{~d} t
\end{aligned}
$$

under variations $(x, d) \mapsto(x+\delta x, d+\delta d)$ satisfying $\operatorname{div}(\delta x)=0$ and $\delta d \cdot d=0$. The last three terms on the right model the dissipative mechanisms. Typically $T_{v}$ is non-symmetric which gives rise to a coupling with 
the angular momentum equation (recall that $\operatorname{rot}(\delta d)$ is skew). The term $C_{v}$ models dissipative interactions between the liquid crystal molecules and is set equal to zero in the classical Ericksen-Leslie equations.

This class of problems admit natural weak statements which inherit the natural energy estimates. Specifically, using the identity

$$
\operatorname{div}\left((\nabla d)^{T}\left[\frac{\partial \mathcal{W}}{\partial \nabla d}\right]\right)=\nabla \mathcal{W}(d, \nabla d)+(\nabla d)^{T}\left(\operatorname{div}\left[\frac{\partial \mathcal{W}}{\partial \nabla d}\right]-\left(\frac{\partial \mathcal{W}}{\partial d}\right)\right)
$$

it follows that

$$
\operatorname{div}\left(T_{e}\right)=\nabla \mathcal{W}(d, \nabla d)+(\nabla d)^{T}\left(\operatorname{div}(C)-g_{e}\right)=\nabla \mathcal{W}(d, \nabla d)+(\nabla d)^{T}\left(\left(\rho \bar{r}^{2}\right) \ddot{d}+\theta d+g_{v}-\rho m\right) .
$$

Absorbing the gradient of $\mathcal{W}$ into the pressure and noting that $(\nabla d)^{T} d=\nabla\left(|d|^{2} / 2\right)=0$ gives the following form of the linear momentum equation:

$$
\rho \dot{v}+\nabla p-\operatorname{div}\left(T_{v}\right)+(\nabla d)^{T}\left(\left(\rho \bar{r}^{2}\right) \ddot{d}+g_{v}-\rho m\right)=\rho f .
$$

The natural weak statements of the momentum equations (assuming homogeneous boundary data) then become

$$
\int_{\Omega} \rho(\dot{v}, w)-(p, \operatorname{div}(w))+\left(T_{v}, \nabla w\right)+\left(\left(\rho \bar{r}^{2}\right) \ddot{d}+g_{v}-\rho m,(\nabla d) w\right)=\int_{\Omega} \rho(f, w),
$$

and

$$
\int_{\Omega}\left(\rho \bar{r}^{2}\right)(\ddot{d}, e)+\left(g_{v}, e\right)+\left(\left(\frac{\partial \mathcal{W}}{\partial d}\right), e\right)+\left(\left[\frac{\partial \mathcal{W}}{\partial \nabla d}\right],(\nabla e)\right)+(\theta d, e)=\int_{\Omega} \rho(m, e) .
$$

Formally selecting $w=v$ and $e=d_{t}$ and assuming $\operatorname{div}(v)=0$ and $|d|=1$ gives the energy estimate

$$
\frac{\mathrm{d}}{\mathrm{d} t} \int_{\Omega}\left((\rho / 2)\left(|v|^{2}+\bar{r}^{2}|\dot{d}|^{2}\right)+\mathcal{W}(d, \nabla d)\right)+\int_{\Omega}\left(T_{v}, \nabla v\right)+\left(g_{v}, \dot{d}\right)=\int_{\Omega}(\rho f, v)+(\rho m . \dot{d}) .
$$

The middle term is the dissipation and the coefficients appearing in equation (1.3) are restricted to guarantee that the integrand is non-negative. Below we assume that $\bar{r} \ll 1$ so that the inertial term in the angular momentum equation can be neglected.

\subsection{Dissipation}

Using equation (1.3) to expand the dissipative term in the energy estimate (2.1) gives

$$
T_{v} \cdot(\nabla v)+\left(g_{v}, \dot{d}\right)=\gamma_{0}\left(d^{T} D(v) d\right)^{2}+\gamma_{1}|\stackrel{d}{d}|^{2}+\mu|D(v)|^{2}+\hat{\gamma}_{2}|D(v) d|^{2}+\left(\hat{\gamma}_{1}+\gamma_{2}\right) \dot{d}^{T} D(v) d .
$$

The coefficients are restricted to guarantee that the term $\left(\hat{\gamma}_{1}+\gamma_{2}\right) \dot{d}^{T} D(v) d$ is dominated by the others.

- The expression $T_{v}:(\nabla v)+g_{v} \cdot \dot{d}$ will be non-negative independently of the constraints $\operatorname{div}(v)=0$ and $|d|=1$ if:

$$
\gamma_{0}, \gamma_{1}, \mu \geq 0, \quad \text { and } \quad \gamma_{1} \hat{\gamma}_{2} \geq(1 / 4)\left(\hat{\gamma}_{1}+\gamma_{2}\right)^{2} .
$$

- When $\operatorname{div}(v)=0$ and $|d|=1$ it follows that $\operatorname{tr}(D(v))=0$ and $\stackrel{d}{d} d=0$. In this situation $T_{v} \cdot(\nabla v)+$ $g_{v} . \dot{d} \geq 0$ whenever, $\gamma_{1}, \mu \geq 0$ and

$$
2 \gamma_{0}+3 \mu+2 \hat{\gamma}_{2} \geq 0, \quad \text { and } \quad \gamma_{1}\left(2 \mu+\hat{\gamma}_{2}\right) \geq\left(\hat{\gamma}_{1}+\gamma_{2}\right)^{2} / 4
$$

Since numerical schemes will not satisfy the constraints it may be necessary to modify to formulae for the dissipative terms to guarantee dissipation. For example, replacing $D(v)$ by $D(v)-(\operatorname{div}(v) / n) I$ or $\stackrel{\AA}{d}$ by $\left(I-d \otimes d /|d|^{2}\right) \dot{d}$. 


\subsection{Boundary conditions}

Below homogeneous Dirichlet boundary data will be assumed for the velocity, $\left.v\right|_{\partial \Omega}=0$, and it will be assumed that $\left.d(t)\right|_{\partial \Omega}=d_{0}$ where $d_{0} \in H^{1}(\Omega)^{n}$ is independent of time. This eliminates the need for translation arguments which are standard but technical [13]. Since all practical flow codes (and experiments) need to accommodate more general boundary conditions we briefly review these in this section.

Consider the situation where $\partial \Omega=\bar{\Gamma}_{0} \cap \bar{\Gamma}_{1}$ with $v=v_{0}$ on $\Gamma_{0}$ and a traction boundary condition on $\Gamma_{1}$ for the linear momentum equation. For stability reasons, we always write

$$
(v \cdot \nabla) v=(1 / 2)((v \cdot \nabla) v+\operatorname{div}(v \otimes v))=(1 / 2)\left(v_{j} v_{i, j}+\left(v_{i} v_{j}\right)_{, j}\right), \text { when } \operatorname{div}(v)=0
$$

Since the elastic part of the stress tensor does not get integrated by parts the natural boundary condition of the weak statement used for the numerical scheme is

$$
\left(-p I+T_{v}-(\rho / 2) v \otimes v\right) n=t .
$$

Similarly, if $\partial \Omega=\bar{\Gamma}_{2} \cap \bar{\Gamma}_{3}$ we may consider boundary data $d=d_{0}$ on $\Gamma_{2}$ and specification of the elastic couple $[\partial \mathcal{W} / \partial \nabla d] n=c$ on $\Gamma_{3}$. The natural weak statement of the Ericksen-Leslie equations is then

$$
\begin{aligned}
& \int_{\Omega}\left\{\rho\left(v_{t}, w\right)+(\rho / 2)(((v . \nabla) v, w)-((v \cdot \nabla) w, v))-(p, \operatorname{div}(w))+\left(T_{v}, \nabla w\right)\right.\left.+\left(g_{v}-\rho m,(\nabla d) w\right)\right\}=\int_{\Omega} \rho(f, w)+\int_{\Gamma_{1}}(t, w) \\
& \int_{\Omega}\left(g_{v}, e\right)+\left(\left(\frac{\partial \mathcal{W}}{\partial d}\right), e\right)+\left(\left[\frac{\partial \mathcal{W}}{\partial \nabla d}\right], \nabla e\right)+(\theta d, e)=\int_{\Omega} \rho(m, e)+\int_{\Gamma_{3}}(c, e)
\end{aligned}
$$

for test functions $(w, e)$ vanishing on $\Gamma_{0} \times \Gamma_{2} ;\left.w\right|_{\Gamma_{0}}=0$, and $\left.e\right|_{\Gamma_{2}}=0$.

When $\left.v\right|_{\Gamma_{0}}=0$ and $\left.d\right|_{\Gamma_{2}}=d_{0}$ is independent of time (presumably with $\left|d_{0}\right|=1$ ), then $(w, e)=\left(v, d_{t}\right)$ is an admissible test function. This choice gives

$$
\frac{\mathrm{d}}{\mathrm{d} t} \int_{\Omega}\left((\rho / 2)|v|^{2}+\mathcal{W}(d, \nabla d)\right)+\int_{\Omega}\left(\left(T_{v}, \nabla v\right)+\left(g_{v} \cdot \dot{d}\right)\right)=\int_{\Omega}(\rho(f, v)+\rho(m, \dot{d}))+\int_{\Gamma_{1}}(t, v)+\int_{\Gamma_{3}}\left(c, d_{t}\right) .
$$

Since $d_{t}$ is not expected to have a trace on $\Gamma_{3}$, it is necessary assume that the boundary couple is differentiable in time and to write

$$
\int_{0}^{T} \int_{\Gamma_{3}}\left(c, d_{t}\right)=\left.\int_{\Gamma_{3}}(c, d)\right|_{0} ^{T}-\int_{0}^{T} \int_{\Gamma_{3}}\left(c_{t}, d\right)
$$

\subsection{Oseen Frank energy}

The classical Oseen-Frank strain energy function is

$$
\begin{aligned}
\mathcal{W}(d, \nabla d)= & \left(k_{1} / 2\right) \operatorname{div}(d)^{2}+\left(k_{2} / 2\right)(d \cdot \operatorname{curl}(d)+q)^{2}+(1 / 2)\left(k_{2}-k_{4}\right)\left(|\nabla d|^{2}-\operatorname{div}(d)^{2}\right. \\
& \left.-|\operatorname{curl}(d)|^{2}\right)+\left(k_{3} / 2\right)|d \times \operatorname{curl}(d)|^{2} .
\end{aligned}
$$

The elastic constants satisfy $k_{i} \geq 0, i=1,2,3$, and $\left|k_{4}\right| \leq k_{2}$, and $q$ is the chiral constant. Table 1 tabulates the elastic stresses and couples that result from each of the terms in this energy. If $k=k_{1}=k_{2}=k_{3}$ and $k_{4}=q=0$ the identity $|\operatorname{curl}(d)|^{2}=(d \cdot \operatorname{curl}(d))^{2}+|d \times \operatorname{curl}(d)|^{2}$ for unit vectors shows $\mathcal{W}(d, \nabla d)=(k / 2)|\nabla d|^{2}$.

The term with coefficient $k_{2}-k_{4}$ is a null Lagrangian (pure divergence) [8,9],

$$
|\nabla d|^{2}-|\operatorname{curl}(d)|^{2}-\operatorname{div}(d)^{2}=\operatorname{tr}\left((\nabla d)^{2}\right)-\operatorname{div}(d)^{2}=\operatorname{div}((\nabla d) d-\operatorname{div}(d) d) .
$$


TABLE 1. Components of the elastic stresses and couples.

\begin{tabular}{|c|c|c|c|}
\hline Energy $\mathcal{W}$ & $C=\partial \mathcal{W} / \partial \nabla d$ & $g_{e}=\partial \mathcal{W} / \partial d$ & $w(d, e)=\langle\delta \mathcal{W} / \delta d, e\rangle$ \\
\hline$(1 / 2)|\nabla d|^{2}$ & $\nabla d$ & 0 & $(\nabla d, \nabla e)$ \\
$(1 / 2) \operatorname{div}(d)^{2}$ & $\operatorname{div}(d) I$ & 0 & $(\operatorname{div}(d), \operatorname{div}(e))$ \\
$d . \operatorname{curl}(d)$ & $\operatorname{rot}(d)$ & $\operatorname{curl}(d)$ & $d \cdot \operatorname{curl}(e)+e \cdot \operatorname{curl}(d)$ \\
$(1 / 2)|\operatorname{curl}(d)|^{2}$ & $2 W(d)$ & 0 & $2(W(d), \nabla e)=2(W(d), W(e))$ \\
$(1 / 2)|d \cdot \operatorname{curl}(d)|^{2}$ & $(d \cdot \operatorname{curl}(d)) \operatorname{rot}(d)$ & $(d \cdot \operatorname{curl}(d)) \operatorname{curl}(d)$ & $(d \cdot \operatorname{curl}(d))(d \cdot \operatorname{curl}(d) d+e \cdot \operatorname{curl}(d))$ \\
$(1 / 2)|d \times \operatorname{curl}(d)|^{2}$ & $4(W(d) d \otimes d)_{\text {skew }}$ & $4 W(d)^{T} W(d) d$ & $4(W(d) d, W(e) d+W(d) e)$ \\
\hline
\end{tabular}

For static variational problems the integral of this term depends only upon the boundary data, so $k_{4}$ can be selected for mathematical convenience [5]. Note, though, that different values of $k_{4}$ will give rise to different natural boundary conditions on the portion of the boundary where $d$ is not specified.

Since numerical approximations only approximate the constraint $|d|=1$ it is necessary to write the energy in a form so that its extension to non-unit vectors preserves essential coercivity properties. For example, when $|d|=1$ the term $d \cdot \operatorname{curl}(d)+q$ may be written as $d \cdot(\operatorname{curl}(d)+q d)$, and the energy may be written as

$$
\begin{aligned}
\mathcal{W}(d, \nabla d)= & \left(k_{1} / 2\right) \operatorname{div}(d)^{2}+(1 / 2) \min \left(k_{2}, k_{3}\right)|\operatorname{curl}(d)+q d|^{2} \\
& +(1 / 2) \max \left(k_{2}-k_{3}, 0\right)(d \cdot(\operatorname{curl}(d)+q d))^{2}+(1 / 2) \max \left(k_{3}-k_{2}, 0\right)|d \times \operatorname{curl}(d)|^{2} \\
& +(1 / 2)\left(k_{2}-k_{4}\right)\left(|\nabla d|^{2}-\operatorname{div}(d)^{2}-|\operatorname{curl}(d)|^{2}\right) .
\end{aligned}
$$

If $0<\left(k_{2}-k_{4}\right) \leq(1 / 2) \min \left(k_{1}, k_{2}, k_{3}\right)$ then there are constants $C_{e}, c_{e}>0$ such that this expression satisfies a Gårding inequality,

for arbitrary functions $d \in H^{1}(\Omega)^{n}$.

$$
W(d, \nabla d) \geq c_{e}|\nabla d|^{2}-C_{e}|d|^{2},
$$

The quartic terms in the Oseen Frank energy give rise to terms of the form $((\nabla d) d,(\nabla d) e)$ in the weak statements of the angular momentum equation. The theory for vector valued problems of this type is in its infancy and technical $[7,30]$. To date there is no existence theory for the Ericksen-Leslie equations for the most general form of the Oseen Frank energy. To circumvent this issue it will be assumed that the elastic energy is quadratic in the director; that is, $k_{2}=k_{3}$.

\subsection{Lin-Liu equation}

The equations

$$
\begin{aligned}
\rho \dot{v}+\nabla p-\operatorname{div}(\mu D(v))+k(\nabla d)^{T} \Delta d & =\rho f, \\
\gamma \dot{d}-k \Delta d+\theta d & =\rho m,
\end{aligned}
$$

have a similar mathematical structure to the Ericksen-Leslie equations and were studied by Lin and Liu [22,25]. The similarity with the Ericksen-Leslie equations is seen upon setting

$$
k_{1}=k_{2}=k_{3}=k, \quad \gamma_{1}=\gamma, \quad \gamma_{0}=\hat{\gamma}_{1}=\gamma_{2}=\hat{\gamma}_{2}=0,
$$

to get

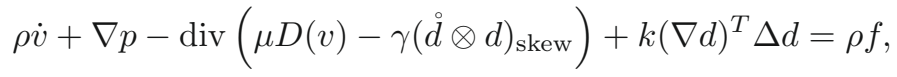

$$
\begin{aligned}
& \gamma \stackrel{\circ}{d}-k \Delta d+\theta d=\rho m,
\end{aligned}
$$

where $\stackrel{d}{d}=\dot{d}-W(v) d$. 
The numerical approximation of solutions to the Lin-Liu equations has been studied extensively $[2,6,13,27-29]$. These papers consider approximations of the weak statement

$$
\begin{array}{r}
\int_{\Omega}(\rho \dot{v}, w)-(p, \operatorname{div}(w))+(\mu D(v), D(w))+(k \Delta d,(w \cdot \nabla) d)=\int_{\Omega}(\rho f, w), \\
\int_{\Omega}(\gamma \dot{d}, e)-(k \Delta d, e)+(\theta d, e)=\int_{\Omega}(\rho m, e),
\end{array}
$$

and an implicit Euler approximation of the time derivative. Since a conforming Galerkin finite element approximations of this weak statement would require Hermite elements, mixed formulations are often employed to circumvent this.

The use of Hermite elements or a mixed method can be avoided using the techniques introduced in Section 2.1 for the Ericksen-Leslie equations. Eliminating $\Delta d$ from the linear momentum equation gives

$$
\rho \dot{v}+\nabla p-\operatorname{div}(\mu D(v))+(\nabla d)^{T}(\gamma \dot{d}-\rho m)=\rho f .
$$

The natural weak statement is then

$$
\begin{aligned}
\int_{\Omega}(\rho \dot{v}, w)-(p, \operatorname{div}(w))+(\mu D(v), D(w))+(\gamma \dot{d}-\rho m,(w \cdot \nabla) d) & =\int_{\Omega}(\rho f, w), \\
\int_{\Omega}(\gamma \dot{d}, e)+(k \nabla d, \nabla e)+(\theta d, e) & =\int_{\Omega} m . e .
\end{aligned}
$$

Selecting $w=v$ and $e=d_{t}$ and adding gives the estimate

$$
(1 / 2) \frac{\mathrm{d}}{\mathrm{d} t} \int_{\Omega}\left(\rho|v|^{2}+k|\nabla d|^{2}\right)+\int_{\Omega}\left(\mu|D(v)|^{2}+\gamma|\dot{d}|^{2}\right)=\int_{\Omega} \rho(f \cdot v+m \cdot \dot{d}) .
$$

Stable and convergent numerical schemes of arbitrary order in time and space were developed by the author in [34] for (penalized) Galerkin approximations of problems having this structure.

\subsection{Penalized weak formulation}

The failure of a satisfactory existence theory for the Ericksen-Leslie equations can be attributed to the constraint $|d|=1$; in particular, the correct space, and estimates, for the associated Lagrange multiplier are not known. In a numerical context this issue is particularly acute $[1,18]$ since "locking" occurs: the only polynomial functions satisfying the constraint $\left|d_{h}\right|=1$ are constants. To circumvent these difficulties the constraint is approximated by allowing $d$ to take values in $\mathbb{R}^{n}$ and adding a penalty term to the energy, $\mathcal{W}_{\epsilon}(d, \nabla d)=\mathcal{W}(d, \nabla d)+(1 / \epsilon) F(d)$, where $F \in C^{1}\left(\mathbb{R}^{n}, \mathbb{R}\right)$ is non-negative and vanishes if and only if $|d|=1$. The prototypical example with quadratic growth is

$$
F(d)=\left\{\begin{array}{cc}
(1 / 2)\left(|d|^{2}-1\right)^{2} & |d| \leq \sqrt{3} \\
2|d|^{2}-4 & |d| \geq \sqrt{3},
\end{array} \quad D F(d)=\left\{\begin{array}{cc}
2\left(|d|^{2}-1\right) d & |d| \leq \sqrt{3} \\
4 d & |d| \geq \sqrt{3}
\end{array}\right.\right.
$$

When the elastic energy is quadratic in $d$, we show that numerical solutions converge to solutions of the following weak statement of the penalized Ericksen-Leslie equations. If the data $d_{0} \in H^{1}(\Omega)^{n}$ satisfies $\left|d_{0}\right| \leq 1$ on $\partial \Omega$, and $v^{0} \in L^{2}(\Omega)^{n}, d^{0} \in d_{0}+H_{0}^{1}(\Omega)^{n}$, then

$$
\begin{aligned}
& v \in\left\{v \in L^{\infty}\left[0, T ; L^{2}(\Omega)\right] \cap L^{2}\left[0, T ; H_{0}^{1}(\Omega)\right] \mid \operatorname{div}(v)=0\right\}, \\
& d-d_{0} \in L^{\infty}\left[0, T ; H_{0}^{1}(\Omega)\right] \cap H^{1}\left[0, T ; L^{4 / 3}(\Omega)\right],
\end{aligned}
$$


satisfy

$$
d(0)=d^{0}, \quad \text { and } \quad d^{T} D(v) d, D(v) d, \stackrel{\circ}{d} \in L^{2}\left[0, T ; L^{2}(\Omega)\right],
$$

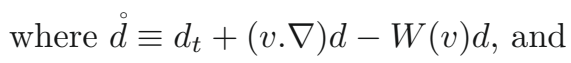

$$
\begin{aligned}
\int_{0}^{T} \int_{\Omega}\left\{-\rho\left(v, w_{t}\right)+(\rho / 2)((v \cdot \nabla) v, w)\right. & -(\rho / 2)((v \cdot \nabla) w, v) \\
+ & \left.\left(T_{v}, \nabla w\right)+\left(g_{v}-\rho m,(\nabla d) w\right)\right\}=\int_{\Omega}\left(v^{0}, w(0)\right)+\int_{0}^{T} \int_{\Omega} \rho(f, w),
\end{aligned}
$$

for all $w \in\left\{w \in L^{2}\left[0, T ; H_{0}^{1}(\Omega)\right] \cap H^{1}\left[0, T ; H^{-1}(\Omega)\right] \mid \operatorname{div}(w)=0\right.$ and $\left.w(T)=0\right\}$ and

$$
\int_{0}^{T} \int_{\Omega}\left(g_{v}, e\right)+\left(\left(\frac{\partial \mathcal{W}}{\partial d}\right), e\right)+\left(\left[\frac{\partial \mathcal{W}}{\partial \nabla d}\right], \nabla e\right)+(1 / \epsilon)(f(d), e)=\int_{0}^{T} \int_{\Omega} \rho(m, e),
$$

for all $e \in L^{2}\left[0, T ; H_{0}^{1}(\Omega)\right]$.

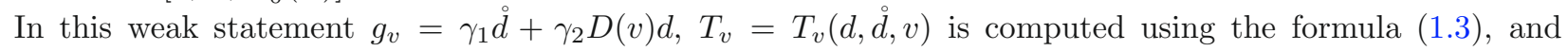
$f(d)=D F(d)$ is the penalty function in equation (2.4).

\section{NumericAl APPROXimation}

\subsection{Introduction}

In order to expose the key ideas involved we restrict the analysis to the following situation. Many of these assumptions can be relaxed using, for example, truncations, regularizations, and various numerical (discrete) tricks; or at the expense of increased technical argument.

Assumption 3.1. The domain, $\Omega \subset \mathbb{R}^{n}$, boundary data, triangulations of the domain, and time partitions, satisfy the following.

1. The domain $\Omega \subset \mathbb{R}^{n}$ is bounded, Lipschitz, and sufficiently regular to admit $H^{2} \times H^{1}$ regularity of the Stokes operator. (It may be possible to relax this condition to $W^{1, p} \times L^{p}$ regularity for some $p>n$.)

2. Homogeneous Dirichlet boundary data is prescribed for the velocity, $\left.v\right|_{\partial \Omega}=0$.

3. Non-homogeneous Dirichlet boundary data, $d_{0} \in H^{1}(\Omega)^{n}$, for the director satisfies $\left|d_{0}\right| \leq 1$ and is independent of time. (Time dependent boundary data can be accommodated using standard, but technical, arguments [13].)

4. Finite element subspaces for the spatial discretization are constructed over quasi-uniform triangulations $\left\{\mathcal{T}_{h}\right\}_{h>0}$ of $\Omega$. (Regular triangulations suffice for most of the arguments; compactness of the director requires quasi-uniform triangulations.)

5. The partitions $0=t_{h}^{0}<t_{h}^{1}<\ldots<t_{h}^{N_{h}}=T$ of $[0, T]$ used for the time stepping are quasi-uniform and $N_{h} \rightarrow \infty$ as $h \rightarrow 0$.

These assumptions are mathematical in nature; however, the following restrictions on the viscous coefficients and elastic energy will limit the class of liquid crystals under consideration.

\section{Assumption 3.2.}

- The Leslie constants satisfy the relations in (2.2) with strict inequality; in particular, there exists a constant $c_{v}>0$

$$
T_{v h}:\left(\nabla v_{h}\right)+g_{v h} \cdot \dot{d}_{h} \geq c_{v}\left(\left|D\left(v_{h}\right)\right|^{2}+\left|d_{h}^{T} D\left(v_{h}\right) d_{h}\right|^{2}+\left|\stackrel{d}{h}_{h}\right|^{2}+\left|D\left(v_{h}\right) d_{h}\right|^{2}\right) .
$$


- The elastic energy is quadratic in d and satisfies the Gärding inequality: there exists constants $c_{e}, C_{e}>0$ such that

$$
\mathcal{W}(d, \nabla d) \geq c_{e}|\nabla d|^{2}-C_{e}|d|^{2} \quad \text { and } \quad\left(\left[\frac{\partial \mathcal{W}}{\partial \nabla d}\right], \nabla d\right)+\left(\left(\frac{\partial \mathcal{W}}{\partial d}\right), d\right) \geq c_{e}|\nabla d|^{2}-C_{e}|d|^{2} .
$$

In particular, if $\mathcal{W}(d, \nabla d)$ is the Oseen Frank energy the quadratic assumption requires $k_{2}=k_{3}$.

The (second order) Crank Nicolson time stepping scheme will be analyzed. An analysis of the (first order) implicit Euler time stepping scheme is identical; however, higher order time stepping schemes encounter additional technicalities ${ }^{3}$.

\subsection{Discrete subspaces}

Let $\left\{\mathcal{T}_{h}\right\}_{h>0}$ be a family of regular triangulations of $\Omega$ and let $V_{h} \subset H_{0}^{1}(\Omega)^{n}, P_{h} \subset L^{2}(\Omega) / \mathbb{R}$ and $D_{h} \subset H_{0}^{1}(\Omega)^{n}$ be finite element subspaces constructed over $\mathcal{T}_{h}$. It will be assumed that the pair $\left(V_{h}, P_{h}\right)$ is div-stable. Denote the discretely divergence free subspaces by

$$
Z_{h}=\left\{v_{h} \in V_{h} \mid \int_{\Omega} \operatorname{div}\left(v_{h}\right) q_{h}=0, q_{h} \in P_{h}\right\} .
$$

To approximate non-homogeneous boundary data for the director, write $D_{h}=\mathcal{D}_{h} \cap H_{0}^{1}(\Omega)^{n}$ where $\mathcal{D}_{h} \subset H^{1}(\Omega)^{n}$ is a finite element subspace constructed on $\mathcal{T}_{h}$ without boundary conditions. For $d_{0} \in H^{1}(\Omega)^{n}$ fixed, let $d_{0 h} \in \mathcal{D}_{h}$ be an interpolant or projection of $d_{0}$. The numerical scheme will seek an approximation $d_{h}$ of the director satisfying $d_{h}(t)-d_{0 h} \in D_{h}$.

In order to construct a fully discrete algorithm we introduce partitions $0=t_{h}^{0}<t_{h}^{1}<\ldots<t_{h}^{N_{h}}=T$ of $[0, T]$ and let

$$
\begin{aligned}
& \mathbb{V}_{h}=\left\{v_{h} \in C\left[0, T ; V_{h}\right]\left|v_{h}\right|_{\left(t_{h}^{n-1}, t_{h}^{n}\right)} \in \mathcal{P}_{1}\left[t_{h}^{n-1}, t_{h}^{n} ; V_{h}\right]\right\}, \\
& \overline{\mathbb{V}}_{h}=\left\{v_{h} \in L^{2}\left[0, T ; V_{h}\right]\left|v_{h}\right|_{\left(t_{h}^{n-1}, t_{h}^{n}\right)} \in \mathcal{P}_{0}\left[t_{h}^{n-1}, t_{h}^{n} ; V_{h}\right]\right\}, \\
& \mathbb{P}_{h}=\left\{p_{h} \in L^{2}\left[0, T ; P_{h}\right]\left|p_{h}\right|_{\left(t_{h}^{n-1}, t_{h}^{n}\right)} \in \mathcal{P}_{0}\left[t_{h}^{n-1}, t_{h}^{n} ; P_{h}\right]\right\}, \\
& \mathbb{D}_{h}=\left\{d_{h} \in C\left[0, T ; D_{h}\right]\left|d_{h}\right|_{\left(t_{h}^{n-1}, t_{h}^{n}\right)} \in \mathcal{P}_{1}\left[t_{h}^{n-1}, t_{h}^{n} ; D_{h}\right]\right\}, \\
& \overline{\mathbb{D}}_{h}=\left\{d_{h} \in L^{2}\left[0, T ; D_{h}\right]\left|d_{h}\right|_{\left(t_{h}^{n-1}, t_{h}^{n}\right)} \in \mathcal{P}_{0}\left[t_{h}^{n-1}, t_{h}^{n} ; D_{h}\right]\right\} .
\end{aligned}
$$

Given $v_{h} \in \mathbb{V}_{h}$ taking values $v_{h}\left(t_{h}^{n}\right)=v^{n}$, let $\bar{v}_{h} \in \overline{\mathbb{V}}_{h}$ denote the piecewise constant (in time) function taking values $\bar{v}_{h}(t)=(1 / 2)\left(v^{n}+v^{n-1}\right)$ on $\left(t_{h}^{n-1}, t_{h}^{n}\right)$; similarly, the projection of $d_{h} \in \mathbb{D}_{h}$ onto $\overline{\mathbb{D}}_{h}$ is denoted by $\bar{d}_{h}$. Elementary properties of these projections, which are local averages, will be used ubiquitously. For example, if $v_{h} \in \mathbb{V}_{h}$, then

$$
\int_{0}^{T}\left(D\left(v_{h}\right), D\left(\bar{w}_{h}\right)\right)=\int_{0}^{T}\left(D\left(\bar{v}_{h}\right), D\left(\bar{w}_{h}\right)\right), \quad \bar{w}_{h} \in \overline{\mathbb{V}}_{h}
$$

\footnotetext{
${ }^{3}$ The discrete energy estimate bounds the (kinetic plus elastic) energy at the partition points $\left\{t^{n}\right\}$. The Crank Nicolson solution is piecewise linear in time so a uniform bound at the partition points bounds the solution at all times; this argument fails for higher order schemes.
} 


\subsection{Numerical scheme}

Assuming boundary data $d_{0 h} \in \mathcal{D}_{h}$, and initial data $v_{h}^{0} \in V_{h}$ and $d_{h}^{0} \in d_{0 h}+D_{h}$ are specified, we seek $\left(v_{h}, p_{h}, d_{h}-d_{h 0}\right) \in \mathbb{V}_{h} \times \mathbb{P}_{h} \times \mathbb{D}_{h}$ such that

$$
\begin{gathered}
\int_{0}^{T} \int_{\Omega}\left\{\rho\left(v_{h t}, \bar{w}_{h}\right)+(\rho / 2)\left(\left(\left(v_{h} . \nabla\right) \bar{v}_{h}, \bar{w}_{h}\right)-\left(\left(v_{h} . \nabla\right) \bar{w}_{h}, \bar{v}_{h}\right)\right)-\left(p_{h}, \operatorname{div}\left(\bar{w}_{h}\right)\right)\right. \\
\left.+\left(T_{v h}, \nabla \bar{w}_{h}\right)+\left(g_{v h}-\rho m,\left(\nabla \bar{d}_{h}\right) \bar{w}_{h}\right)\right\}=\int_{0}^{T} \int_{\Omega} \rho\left(f, \bar{w}_{h}\right), \\
\int_{0}^{T} \int_{\Omega}\left(\operatorname{div}\left(\bar{v}_{h}\right), q_{h}\right)=0, \\
\int_{0}^{T} \int_{\Omega}\left(g_{v h}, \bar{e}_{h}\right)+\left(\left(\frac{\partial \mathcal{W}_{h}}{\partial d_{h}}\right), \bar{e}_{h}\right)+\left(\left[\frac{\partial \mathcal{W}_{h}}{\partial \nabla d_{h}}\right], \nabla \bar{e}_{h}\right)+(1 / \epsilon)\left(f\left(d_{h}\right), \bar{e}_{h}\right)=\int_{0}^{T} \int_{\Omega} \rho\left(m, \bar{e}_{h}\right),
\end{gathered}
$$

for all $\left(\bar{w}_{h}, q_{h}, \bar{e}_{h}\right) \in \overline{\mathbb{V}}_{h} \times \mathbb{P}_{h} \times \overline{\mathbb{D}}_{h}$. The convected derivative of the director and energy are calculated as

$$
\stackrel{\circ}{d}_{h}=\dot{d}_{h}-W\left(\bar{v}_{h}\right) d_{h} \equiv d_{h t}+\left(\bar{v}_{h} \cdot \nabla\right) \bar{d}_{h}-W\left(\bar{v}_{h}\right) d_{h}, \quad \text { and } \quad \mathcal{W}_{h} \equiv \mathcal{W}\left(d_{h}, \nabla d_{h}\right),
$$

and the viscous terms are computed as

$$
g_{v h}=\gamma_{1} \stackrel{\circ}{d}_{h}+\gamma_{2} D\left(\bar{v}_{h}\right) d_{h}, \quad \text { and } \quad T_{v h}=T_{v}\left(d_{h}, \stackrel{\circ}{d}_{h}, \bar{v}_{h}\right),
$$

where $T_{v}(., .,$.$) is given by the formula (1.3).$

For the Crank Nicolson time stepping scheme used here, the discrete energy estimate bounds gradients of the projections $\bar{v}_{h}$ and $\bar{d}_{h}$ of the velocity and director respectively. This was why gradients of these projections were used in the nonlinear terms. Notice too that

$$
\int_{0}^{T} \int_{\Omega}\left(\left(v_{h} . \nabla\right) \bar{v}_{h}, \bar{w}_{h}\right)-\left(\left(v_{h} . \nabla\right) \bar{w}_{h}, \bar{v}_{h}\right)=\int_{0}^{T} \int_{\Omega}\left(\left(\bar{v}_{h} . \nabla\right) \bar{v}_{h}, \bar{w}_{h}\right)-\left(\left(\bar{v}_{h} \cdot \nabla\right) \bar{w}_{h}, \bar{v}_{h}\right),
$$

and since the elastic energy is assumed to be quadratic in $d$, the expression

$$
w(d, e) \equiv \int_{\Omega}\left(\left(\frac{\partial \mathcal{W}}{\partial d}\right), e\right)+\left(\left[\frac{\partial \mathcal{W}}{\partial \nabla d}\right], \nabla e\right)
$$

is bilinear. In particular, if $d_{h} \in \mathbb{D}_{h}$ then

$$
\int_{0}^{T} w\left(d_{h}, \bar{e}_{h}\right)=\int_{0}^{T} w\left(\bar{d}_{h}, \bar{e}_{h}\right), \quad \bar{e}_{h} \in \overline{\mathbb{D}}_{h}
$$

\subsection{Stability}

Setting $\left(\bar{w}_{h}, q_{h}, \bar{e}_{h}\right)=\left(\bar{v}_{h}, p_{h}, d_{h t}\right)$ in the discrete weak statement (3.1) gives for each $1 \leq n \leq N$

$$
\begin{aligned}
E\left(v^{n}, d^{n} ; \epsilon\right)+c_{v} \int_{0}^{t_{h}^{n}}\left\{\left\|D\left(\bar{v}_{h}\right)\right\|_{L^{2}(\Omega)}^{2}+\left\|d_{h}^{T} D\left(\bar{v}_{h}\right) d_{h}\right\|_{L^{2}(\Omega)}^{2}+\right. & \left.\left\|\dot{d}_{h}\right\|_{L^{2}(\Omega)}^{2}+\left\|D\left(\bar{v}_{h}\right) d_{h}\right\|_{L^{2}(\Omega)}^{2}\right\} \\
& \leq E\left(v_{h}^{0}, d_{h}^{0} ; \epsilon\right)+\int_{0}^{t_{h}^{n}} \int_{\Omega}\left(\rho f, v_{h}\right)+\left(\rho m, \dot{d}_{h}\right) .
\end{aligned}
$$


In this expression

$$
E(v, d ; \epsilon)=(\rho / 2)\|v\|_{L^{2}(\Omega)}^{2}+\int_{\Omega}\left(\mathcal{W}(d)+3 C_{e}\right)+(1 / \epsilon)\|F(d)\|_{L^{1}(\Omega)}
$$

is the sum of the kinetic and elastic energies, and we have used Assumption 3.2 to estimate the viscous terms. The constant term $3 C_{e}$ is introduced for convenience; the energy is non-negative whenever $\epsilon<1 / C_{e}$.

In the absence of a maximum principle, the term $\left(m, \dot{d}_{h}\right)=\left(m, \dot{d}_{h}+W(\bar{v}) d_{h}\right)$ on the right of equation (3.2) is not immediately bounded by terms on the left. The following lemma shows that this difficulty can be mitigated by selecting the penalty parameter to be sufficiently small (independently of $h$ ).

Lemma 3.1. Let the elastic energy and dissipative terms satisfy Assumption 3.2 and assume that the boundary values for the director satisfy $\left|d_{0 h}\right| \leq \sqrt{3}$. Then there exists a constant $C \geq 0$ depending upon the viscous and elastic constants such that $C\|m\|_{L^{2}\left[0, T ; L^{4}(\Omega)\right]}^{4} \leq 1 / \epsilon$ guarantees the approximate solutions computed using the discrete weak statement (3.1) satisfy

$$
v_{h} \in L^{\infty}\left[0, T ; L^{2}(\Omega)\right], \quad d_{h}, \bar{d}_{h} \in L^{\infty}\left[0, T ; H^{1}(\Omega)\right], \quad(1 / \epsilon) F\left(d^{n}\right) \in L^{1}(\Omega),
$$

and

$$
\bar{v}_{h} \in L^{2}\left[0, T ; H_{0}^{1}(\Omega)\right], \quad \stackrel{\circ}{d}_{h}, d_{h}^{T} D\left(\bar{v}_{h}\right) d_{h}, D\left(\bar{v}_{h}\right) d_{h} \in L^{2}\left[0, T ; L^{2}(\Omega)\right],
$$

with norms bounded by constants depending only upon the initial energy, $E\left(v_{h}^{0}, d_{h}^{0} ; \epsilon\right)$, and the norms $\|f\|_{L^{2}\left[0, T ; H^{-1}(\Omega)\right]}$ and $\|m\|_{L^{2}\left[0, T ; L^{2}(\Omega)\right]}$ of the data.

Remark 3.1. For simplicity we required $m \in L^{2}\left[0, T ; L^{4}(\Omega)\right]$. The same argument using the Sobolev embedding theorem is applicable if $m \in L^{2}\left[0, T ; L^{p}(\Omega)\right]$ for any $p>n$.

Proof. If $\epsilon<1 / C_{e}$ then the energy is non-negative; specifically, Assumption 3.2 bounds the energy below as

$$
E(v, d ; \epsilon) \geq(\rho / 2)\|v\|_{L^{2}(\Omega)}+c_{e}\|\nabla d\|_{L^{2}(\Omega)}-C_{e}\|d\|_{L^{2}(\Omega)}+(1 / \epsilon)\|F(d)\|_{L^{1}(\Omega)}+3 C_{e}|\Omega|,
$$

and using the formula (2.4) for $F(d)$ we find

$$
\begin{aligned}
(1 / \epsilon) F(d)-C_{e}|d|^{2} & = \begin{cases}(1 / \epsilon) F(d)-C_{e}|d|^{2} & |d| \leq \sqrt{3} \\
(1 / 2 \epsilon) F(d)+\left(1 / \epsilon-C_{e}\right)|d|^{2}-(2 / \epsilon) & |d| \geq \sqrt{3}\end{cases} \\
& \geq(1 / 2 \epsilon) F(d)-3 C_{e}
\end{aligned}
$$

We need to bound the last term in the energy estimate (3.2) by the terms on the left. Bounding $\dot{d}_{h}$ as

$$
\left|\dot{d}_{h}\right| \leq\left|\stackrel{\circ}{d}_{h}\right|+\left|W\left(\bar{v}_{h}\right)\right|\left|d_{h}\right| \leq\left|\stackrel{\circ}{d}_{h}\right|+\left|W\left(\bar{v}_{h}\right)\right|\left(\sqrt{3}+\left(\left|d_{h}\right|-\sqrt{3}\right)_{+}\right)
$$

and using the Cauchy-Schwarz, Korn, and Poincaré inequalities it follows that

$$
\begin{aligned}
& E\left(v^{n}, d^{n} ; \epsilon\right)+c \int_{0}^{t_{h}^{n}}\left\{\left\|D\left(\bar{v}_{h}\right)\right\|_{L^{2}(\Omega)}^{2}+\left\|d_{h}^{T} D\left(\bar{v}_{h}\right) d_{h}\right\|_{L^{2}(\Omega)}^{2}+\left\|\stackrel{\circ}{d}_{h}\right\|_{L^{2}(\Omega)}^{2}+\left\|D\left(\bar{v}_{h}\right) d_{h}\right\|_{L^{2}(\Omega)}^{2}\right\} \\
& \quad \leq E\left(v_{h}^{0}, d_{h}^{0} ; \epsilon\right)+C \int_{0}^{t_{h}^{n}}\left\{\|f\|_{H^{-1}(\Omega)}^{2}+\|m\|_{L^{2}(\Omega)}^{2}+\|m\|_{L^{4}(\Omega)}\left\|D\left(\bar{v}_{h}\right)\right\|_{L^{2}(\Omega)}\left\|\left(\left|d_{h}\right|-\sqrt{3}\right)_{+}\right\|_{L^{4}(\Omega)}\right\}
\end{aligned}
$$

Under the assumption that $\left|d_{0 h}\right| \leq \sqrt{3}$ on the boundary, it follows that $\left(\left|d_{h}\right|-\sqrt{3}\right)_{+} \in H_{0}^{1}(\Omega)$ with gradient $\operatorname{sgn}_{+}\left(\left|d_{h}\right|-\sqrt{3}\right)\left(\nabla d_{h}\right)^{T}\left(d_{h} /\left|d_{h}\right|\right)$. The Sobolev embedding theorem (in three dimensions) then shows

$$
\left\|\left(d_{h}-\sqrt{3}\right)_{+}\right\|_{L^{4}(\Omega)} \leq C\left\|\left(d_{h}-\sqrt{3}\right)_{+}\right\|_{L^{2}(\Omega)}^{1 / 4}\|\nabla d\|_{L^{2}(\Omega)}^{3 / 4} .
$$


Using the fact that $F(d) \geq(|d|-\sqrt{3})_{+}^{2}$, the Young inequality shows that for all $\eta>0$ there exists $C_{\eta}>0$ such that

$$
\begin{aligned}
\int_{0}^{t_{h}^{n}} C\|m\|_{L^{4}(\Omega)}\left\|D\left(\bar{v}_{h}\right)\right\|_{L^{2}(\Omega)}\left\|\left(\left|d_{h}\right|-\sqrt{3}\right)_{+}\right\|_{L^{4}(\Omega)} \\
\leq C\|m\|_{L^{2}\left[0, t^{n} ; L^{4}(\Omega)\right]}\left\|D\left(\bar{v}_{h}\right)\right\|_{L^{2}\left[0, t^{n} ; L^{2}(\Omega)\right]}\left\|\left(\left|d_{h}\right|-\sqrt{3}\right)_{+}\right\|_{L^{\infty}\left[0, t^{n} ; L^{4}(\Omega)\right]} \\
\leq \eta\left\|D\left(\bar{v}_{h}\right)\right\|_{L^{2}\left[0, t^{n} ; L^{2}(\Omega)\right]}^{2}+(1 / 2) \max _{1 \leq m \leq n}\left(c_{e}\left\|\nabla d^{m}\right\|_{L^{2}(\Omega)}^{2}+C_{\eta}\|m\|_{L^{2}\left[0, t^{n} ; L^{4}(\Omega)\right]}^{4}\left\|F\left(d^{m}\right)\right\|_{L^{1}(\Omega)}\right) .
\end{aligned}
$$

By selecting $1 \leq n \leq N_{h}$ in equation (3.3) where $\max _{1 \leq n \leq N_{h}}\left(c_{e}\left\|\nabla d^{n}\right\|_{H^{1}(\Omega)}^{2}+(1 / \epsilon)\left\|F\left(d^{n}\right)\right\|_{L^{1}(\Omega)}\right)$ is achieved, the above shows that the last term is dominated by $E\left(v^{n}, d^{n} ; \epsilon\right)$ when $\epsilon$ is sufficiently small, and the lemma follows.

The bound on $\stackrel{\circ}{h}_{h}$ facilitates an estimate of $d_{h t}$ which will used to established compactness of the sequence $\left\{\bar{d}_{h}\right\}_{h>0}$.

Corollary 3.1. Under the hypothesis of the lemma there exists a constant $C>0$ independent of $\epsilon$ and $h$ such that

$$
\left\|d_{h t}\right\|_{L^{2}\left[0, T ; L^{3 / 2}(\Omega)\right]} \leq C .
$$

Proof. From the definition of $\stackrel{\circ}{h}_{h}$ we have $d_{h t}=\stackrel{\circ}{d}_{h}-\left(\bar{v}_{h} . \nabla\right) \bar{d}_{h}+W\left(\bar{v}_{h}\right) d_{h}$. Using the Holder inequality it follows that

$$
\left\|d_{h t}\right\|_{L^{3 / 2}(\Omega)} \leq\left\|\stackrel{d}{h}_{h}\right\|_{L^{3 / 2}(\Omega)}+\left\|\bar{v}_{h}\right\|_{L^{6}(\Omega)}\left\|\nabla \bar{d}_{h}\right\|_{L^{2}(\Omega)}+\left\|W\left(\bar{v}_{h}\right)\right\|_{L^{2}(\Omega)}\left\|d_{h}\right\|_{L^{6}(\Omega)} .
$$

In three or less dimensions $H^{1}(\Omega) \hookrightarrow L^{6}(\Omega)$, and since $d_{h} \in L^{\infty}\left[0, T ; H^{1}(\Omega)\right]$ and $v_{h} \in L^{2}\left[0, T ; H_{0}^{1}(\Omega)\right]$ the estimate follows.

This stability estimate remains valid for the full (non-convex) Ericksen-Leslie energy (i.e. with $k_{2} \neq k_{3}$ ) provided $d_{h}$ is used in place of $\bar{d}_{h}$. This is not so for the following corollary which exploits linearity in an essential fashion.

Corollary 3.2. Under the assumptions of the lemma, there exists $G_{h} \in L^{2}\left[0, T ; L^{2}(\Omega)\right]$ with $\left\|G_{h}\right\|_{L^{2}\left[0, T ; L^{2}(\Omega)\right]} \leq$ $C(\epsilon)$ (and the data for the problem), such that

$$
\int_{0}^{T} \int_{\Omega}\left(\left(\frac{\partial \mathcal{W}_{h}}{\partial d_{h}}\right), \bar{e}_{h}\right)+\left(\left[\frac{\partial \mathcal{W}_{h}}{\partial \nabla d_{h}}\right], \nabla \bar{e}_{h}\right)+(4 / \epsilon)\left(\bar{d}_{h}, \bar{e}_{h}\right)=\int_{0}^{T} \int_{\Omega}\left(G_{h}, \bar{e}_{h}\right)
$$

for all $\bar{e}_{h} \in \overline{\mathbb{D}}_{h}$.

Proof. Write the third equation in (3.1) as

$$
\begin{aligned}
\int_{0}^{T} \int_{\Omega}\left(\left(\frac{\partial \mathcal{W}_{h}}{\partial d_{h}}\right), \bar{e}_{h}\right)+\left(\left[\frac{\partial \mathcal{W}_{h}}{\partial \nabla d_{h}}\right], \nabla \bar{e}_{h}\right)+(4 / \epsilon)\left(\bar{d}_{h}, \bar{e}_{h}\right) & \\
& =\int_{0}^{T} \int_{\Omega} \rho\left(m, \bar{e}_{h}\right)-\left(g_{v h}, \bar{e}_{h}\right)+(1 / \epsilon)\left(4 d_{h}-f\left(d_{h}\right), \bar{e}_{h}\right),
\end{aligned}
$$

for all $\bar{e}_{h} \in \overline{\mathbb{D}}_{h}$. The term $(4 / \epsilon)\left(d_{h}, \bar{e}_{h}\right)$ has been introduced to both sides, and since it is paired with $\bar{e}_{h} \in \overline{\mathbb{D}}_{h}$ was written in terms of the local average $\bar{d}_{h}$ on the left.

Then $m \in L^{2}\left[0, T ; L^{2}(\Omega)\right]$, and the lemma shows $g_{v h} \in L^{2}\left[0, T ; L^{2}(\Omega)\right]$. The remaining term on the right is bounded since

$$
4 d-f(d)=\left\{\begin{array}{cc}
2\left(3-|d|^{2}\right) d & |d| \leq \sqrt{3} \\
0 & |d| \geq \sqrt{3}
\end{array} \quad \text { so } \quad|4 d-f(d)| \leq 4\right.
$$




\subsection{Compactness}

In this section we establish compactness of the velocity and director in $L^{2}\left[0, T ; L^{2}(\Omega)\right]$ and $L^{2}\left[0, T ; H^{1}(\Omega)\right]$ respectively.

\subsubsection{Compactness of the velocity}

The following lemma from [34], Lemma 4.7 , shows that a bound on the derivative of $v_{h}$ is sufficient to obtain equicontinuity of the projection $\bar{v}_{h}$.

Lemma 3.2. Let $\ell \geq 1$ be an integer and $0=t^{0}<t^{1}<\ldots<t^{N}=T$ be a partition of $[0, T]$ and let $\tau=\max _{1 \leq n \leq N}\left(t^{n}-t^{n-1}\right)$ and $\vartheta=\min _{1 \leq n \leq N}\left(t^{n}-t^{n-1}\right) / \tau$. Let $W$ be a (semi) inner product space, $1 \leq q \leq \infty$, and suppose that

$$
w \in\left\{C[0, T ; W]|w|_{\left(t^{n-1}, t^{n}\right)} \in \mathcal{P}_{\ell}\left[t^{n-1}, t^{n} ; W\right] 1 \leq n \leq N\right\},
$$

and that $w^{\prime} \in L^{q}[0, T ; W]$. Then there exists a constant $C=C(\ell, \vartheta)$ depending only upon $\ell$ and $\vartheta$ such that

$$
\left(\int_{\delta}^{T}\|\bar{w}(t)-\bar{w}(t-\delta)\|_{W}^{q} d t\right)^{1 / q} \leq C\left\|w^{\prime}\right\|_{L^{q}[0, T ; W]} \max (\delta, \tau)^{1 / q^{\prime}} \delta^{1 / q},
$$

where $\bar{w}$ is the projection of $w$ onto $\mathcal{P}_{\ell-1}[0, T ; W]$.

The following lemma will be used to bound the time derivatives of the velocities in a certain dual space.

Lemma 3.3. Let $\Omega \subset \mathbb{R}^{n}$ be bounded and sufficiently regular to admit $H^{2} \times H^{1}$ regularity of the Stokes operator. Let $\left(V_{h}, P_{h}\right)$ be a classical div-stable finite element pair constructed over a quasi-uniform triangulation $\mathcal{T}_{h}$ of $\Omega$, and let $Z_{h} \subset V_{h}$ be the discretely divergence free subspace. If $0 \leq n(1 / 2-1 / p) \leq 1$ there exists a constant $C>0$ such that the orthogonal projection $P_{h}: L^{2}(\Omega) \rightarrow Z_{h}$ satisfies

$$
\left\|P_{h} w\right\|_{W^{1, p}(\Omega)} \leq C\|w\|_{H^{2}(\Omega)}, \quad w \in W \equiv\left\{w \in H^{2}(\Omega) \cap H_{0}^{1}(\Omega) \mid \operatorname{div}(w)=0\right\} .
$$

Remark 3.2. In a pde context technical difficulties with the projection $P_{h}$ can be avoided by selecting the subspaces for the velocity to be the first $N$ eigenfunctions of the Stokes operator in $W=\left\{w \in H_{0}^{1}(\Omega) \cap H^{2}(\Omega) \mid\right.$ $\operatorname{div}(w)=0\}$. Expanding the functions in $W$ using this eigenbasis immediately shows that the projections are bounded.

Proof. Let $\Pi_{h}: H_{0}^{1}(\Omega) \rightarrow V_{h}$ be the Stokes projection; that is, $w_{h}=\Pi_{h} w$ satisfies

$$
w_{h} \in Z_{h}, \quad \int_{\Omega}\left(D\left(w_{h}\right), D\left(z_{h}\right)\right)=\int_{\Omega}\left(D(w), D\left(z_{h}\right)\right), \quad z_{h} \in Z_{h} .
$$

The Aubin-Nitsche duality argument shows $\left\|w-\Pi_{h} w\right\|_{L^{2}(\Omega)} \leq C h^{2}\|w\|_{H^{2}(\Omega)}$ for divergence free functions $w \in H^{2}(\Omega) \cap H_{0}^{1}(\Omega)$; moreover, it was shown in [14] that there exists a constant $C>0$ such that $\left\|\Pi_{h} w\right\|_{W^{1, p}(\Omega)} \leq$ $C\|w\|_{W^{1, p}(\Omega)}$. Combining this with classical inverse inequalities shows

$$
\begin{aligned}
\left\|P_{h} w\right\|_{W^{1, p}(\Omega)} & \leq\left\|\left(P_{h}-\Pi_{h}\right) w\right\|_{W^{1, p}(\Omega)}+\left\|\Pi_{h} w\right\|_{W^{1, p}(\Omega)} \\
& \leq \frac{C}{h^{1+n(1 / 2-1 / p)}}\left\|\left(P_{h}-\Pi_{h}\right) w\right\|_{L^{2}(\Omega)}+C\|w\|_{W^{1, p}(\Omega)} \\
& \leq \frac{C}{h^{1+n(1 / 2-1 / p)}}\left\|w-\Pi_{h} w\right\|_{L^{2}(\Omega)}+C\|w\|_{H^{2}(\Omega)} \\
& \leq \frac{C h^{2}}{h^{1+n(1 / 2-1 / p)}}\|w\|_{H^{2}(\Omega)}+C\|w\|_{H^{2}(\Omega)} .
\end{aligned}
$$


Compactness of the velocities will first be established in $W^{\prime}$, where $W=\left\{w \in H_{0}^{1}(\Omega) \cap H^{2}(\Omega) \mid \operatorname{div}(w)=0\right\}$. Since $W$ is not dense in $L^{2}(\Omega)$, the natural mapping $\iota: H_{0}^{1}(\Omega) \rightarrow W^{\prime}$ given by $\iota(v)(w)=(v, w)_{L^{2}(\Omega)}$ is not injective; the kernel is characterized by [12], Lemma III.1.1,

$$
\begin{aligned}
K(\iota) & =\left\{v \in H_{0}^{1}(\Omega) \mid(v, w)_{L^{2}(\Omega)}=0, w \in W\right\} \\
& =H_{0}^{1}(\Omega) \cap\left\{\nabla \phi \mid \phi \in W_{\text {loc }}^{1,1}(\Omega)\right\} \\
& =\left\{\nabla \phi\left|\phi \in H^{2}(\Omega), \partial \phi / \partial n\right|_{\partial \Omega}=0, \text { and }\left.\phi\right|_{\Gamma_{i}} \in \mathbb{R}\right\}
\end{aligned}
$$

where $\left\{\Gamma_{i}\right\}$ are the components of $\partial \Omega$. The Lions-Aubin theorem will show $\left\{\iota\left(v_{h}\right)\right\}_{h>0}$ is compact in $L^{p}\left[0, T ; W^{\prime}\right]$. Compactness in $L^{p}\left[0, T ; L^{2}(\Omega)\right]$ will follow from the following lemma.

Lemma 3.4. Let $\Omega \subset \mathbb{R}^{n}$ be a bounded Lipschitz domain and $\left\{\left(V_{h}, P_{h}\right)\right\}_{h>0}$ be a classical family of div-stable finite element spaces constructed over a family of regular triangulations of $\Omega$. Let $\left\{Z_{h}\right\}_{h>0}$ denote the family of discretely divergence free spaces and $W=\left\{w \in H_{0}^{1}(\Omega) \cap H^{2}(\Omega) \mid \operatorname{div}(w)=0\right\}$ and $\iota: H_{0}^{1}(\Omega) \rightarrow W^{\prime}$ be the natural mapping: $\iota(v)(w)=(v, w)_{L^{2}(\Omega)}$. Then for each $\epsilon>0$ there exists $C(\epsilon)>0$ such that

$$
\left\|v_{h}\right\|_{L^{2}(\Omega)} \leq \epsilon\left\|v_{h}\right\|_{H^{1}(\Omega)}+C(\epsilon)\left\|\iota\left(v_{h}\right)\right\|_{W^{\prime}}
$$

for every set $\left\{v_{h}\right\}_{h \searrow 0} \subset H_{0}^{1}(\Omega)$ with $v_{h} \in Z_{h}$.

Proof. Arguing by contradiction, assume there exists $\epsilon>0$ and a sequence $v_{n} \in Z_{h_{n}}$ with $h_{n} \searrow 0$ such that $\left\|v_{n}\right\|_{L^{2}(\Omega)}=1$ and

$$
1=\left\|v_{n}\right\|_{L^{2}(\Omega)}>\epsilon\left\|v_{n}\right\|_{H^{1}(\Omega)}+n\left\|\iota\left(v_{n}\right)\right\|_{W^{\prime}} .
$$

Compactness of the embedding $H^{1}(\Omega) \hookrightarrow L^{2}(\Omega)$ and continuity of $\iota$ allows passage to a subsequence for which

$$
\begin{aligned}
v_{n} & \rightarrow v & & \text { in } H_{0}^{1}(\Omega) \\
v_{n} & \rightarrow v & & \text { in } L^{2}(\Omega) \\
\iota\left(v_{n}\right) & \rightarrow 0=\iota(v) & & \text { in } W^{\prime} .
\end{aligned}
$$

The last property shows $v \in \operatorname{Kerr}(\iota)$, so $v=\nabla \phi$ for some $\phi \in H^{2}(\Omega)$ with $\partial \phi / \partial n=0$ on $\partial \Omega$. Also, given $p \in L^{2}(\Omega)$, let $p_{n} \in P_{h_{n}}$, be a sequence for which $p_{n} \rightarrow p$ in $L^{2}(\Omega)$. Since $\operatorname{div}\left(v_{h}\right) \rightarrow \operatorname{div}(v)$ in $L^{2}(\Omega)$ it follows that

$$
\int_{\Omega} \operatorname{div}(v) p=\lim _{n} \int_{\Omega} \operatorname{div}\left(v_{n}\right) p_{n}=0
$$

so $\Delta \phi=\operatorname{div}(v)=0$. It follows that $\phi$ is constant on each component of $\Omega$, so $v=\nabla \phi=0$. This establishes the contradiction:

$$
1=\left\|v_{h}\right\|_{L^{2}(\Omega)} \rightarrow\|v\|_{L^{2}(\Omega)}=0 .
$$

Lemma 3.5. Let the domain, boundary data, and space and time partitions satisfy Assumptions 3.1. Assume $\left\{\left(V_{h}, P_{h}\right)\right\}_{h>0}$ is a family of classical div-stable finite element pairs and let the hypotheses of Lemma 3.1 hold. Then the velocities $\left\{\bar{v}_{h}\right\}_{h>0}$ are compact in $L^{p}\left[0, T ; L^{2}(\Omega)\right]$ for $1 \leq p<\infty$.

Proof. Restricting the test functions $\bar{w}_{h}$ in equation $(3.1)_{1}$ to take values in the discretely divergence free space, $Z_{h}$, shows

$$
\begin{aligned}
& \int_{\Omega} \rho\left(v_{h t}, \bar{w}_{h}\right)=\int_{\Omega}\left\{\rho\left(f, \bar{w}_{h}\right)-(\rho / 2)\left(\left(\left(v_{h} \cdot \nabla\right) \bar{v}_{h}, \bar{w}_{h}\right)-\left(\left(v_{h} \cdot \nabla\right) \bar{w}_{h}, \bar{v}_{h}\right)\right)\right. \\
&\left.-\left(T_{v h}, \nabla \bar{w}_{h}\right)-\left(g_{v h}-\rho m,\left(\nabla \bar{d}_{h}\right) \bar{w}_{h}\right)\right\} .
\end{aligned}
$$


A calculation using the Sobolev embedding theorem (in three or less dimensions) shows

$$
\begin{aligned}
& \int_{0}^{T} \int_{\Omega}\left\{\rho\left(f, \bar{w}_{h}\right)-(\rho / 2)\left(\left(\left(v_{h} \cdot \nabla\right) \bar{v}_{h}, \bar{w}_{h}\right)-\left(\left(v_{h} \cdot \nabla\right) \bar{w}_{h}, \bar{v}_{h}\right)\right)\right\} \\
& \leq C\left(\|f\|_{L^{2}\left[0, T ; H^{-1}(\Omega)\right]}+\left\|\bar{v}_{h}\right\|_{L^{\infty}\left[0, T ; L^{2}(\Omega)\right]}^{1 / 2}\left\|\nabla \bar{v}_{h}\right\|_{L^{2}\left[0, T ; L^{2}(\Omega)\right]}^{3 / 2}\left\|\nabla \bar{w}_{h}\right\|_{L^{4}\left[0, T ; L^{2}(\Omega)\right]}\right.
\end{aligned}
$$

The most singular term in the viscous stress, $T_{v h}$, is the one with coefficient $\gamma_{0}$, and may be bounded as

$$
\begin{aligned}
\int_{0}^{T} \int_{\Omega}\left(d_{h}^{T} D\left(\bar{v}_{h}\right) d_{h}\right)\left(d_{h}^{T} D\left(\bar{w}_{h}\right) d_{h}\right) & \leq \int_{0}^{T}\left\|d_{h}^{T} D\left(\bar{v}_{h}\right) d_{h}\right\|_{L^{2}(\Omega)}\left\|d_{h}\right\|_{L^{6}}^{2}\left\|\nabla \bar{w}_{h}\right\|_{L^{6}(\Omega)} \\
& \leq\left\|d_{h}^{T} D\left(\bar{v}_{h}\right) d_{h}\right\|_{L^{2}\left[0, T ; L^{2}(\Omega)\right]}\left\|d_{h}\right\|_{L^{\infty}\left[0, T ; H^{1}(\Omega)\right]}^{2}\left\|\nabla \bar{w}_{h}\right\|_{L^{2}\left[0, T ; L^{6}(\Omega)\right]}
\end{aligned}
$$

Similarly, the viscous coupling term is bounded as

$$
\begin{aligned}
\int_{0}^{T} \int_{\Omega}\left(g_{v h},\left(\nabla \bar{d}_{h}\right) \bar{w}_{h}\right) & \leq \int_{0}^{T}\left\|g_{v h}\right\|_{L^{2}(\Omega)}\left\|\nabla \bar{d}_{h}\right\|_{L^{2}(\Omega)}\left\|\bar{w}_{h}\right\|_{L^{\infty}(\Omega)} \\
& \leq C\left\|g_{v h}\right\|_{L^{2}\left[0, T ; L^{2}(\Omega)\right]}\left\|\bar{d}_{h}\right\|_{L^{\infty}\left[0, T ; H^{1}(\Omega)\right]}\left\|\nabla \bar{w}_{h}\right\|_{L^{2}\left[0, T ; L^{4}(\Omega)\right]}
\end{aligned}
$$

Combining the above estimates with the bounds guaranteed by Lemma 3.1 shows that there exists a constant $C>0$, independent of $h$ and $\epsilon$, for which

$$
\int_{0}^{T} \int_{\Omega}\left(v_{h t}, \bar{w}_{h}\right) \leq C\left\|\bar{w}_{h}\right\|_{L^{4}\left[0, T ; W^{1,6}(\Omega)\right]}, \quad \bar{w}_{h} \in \overline{\mathbb{V}}_{h} \cap L^{2}\left[0, T ; Z_{h}\right] .
$$

Let $W=\left\{w \in H_{0}^{1}(\Omega) \cap H^{2}(\Omega) \quad \operatorname{div}(w)=0\right\}$, and $P_{h}: L^{2}(\Omega) \rightarrow Z_{h}$ be the orthogonal projection. If $w \in L^{4}[0, T ; W]$, let $\bar{w}(t)$ denote the piecewise constant function (in time) taking the average value of $w$ on each interval $\left(t_{h}^{n-1}, t_{h}^{n}\right)$ of the partition. Then $P_{h} \bar{w} \in \overline{\mathbb{V}}_{h}$ takes values in $Z_{h}$, and using Lemma 3.3 (with $p=6$ ) we obtain

$$
\begin{aligned}
\int_{0}^{T} \int_{\Omega}\left(v_{h t}, w\right) & =\int_{0}^{T} \int_{\Omega}\left(v_{h t}, P_{h} \bar{w}\right) \\
& \leq C\left\|P_{h} \bar{w}\right\|_{L^{4}\left[0, T ; W^{1,6}(\Omega)\right]} \\
& \leq C\|\bar{w}\|_{L^{4}\left[0, T ; H^{2}(\Omega)\right]} \\
& \leq C\|w\|_{L^{4}\left[0, T ; H^{2}(\Omega)\right]}, \quad w \in L^{4}[0, T ; W] .
\end{aligned}
$$

It follows that $\left\{\iota\left(v_{h t}\right)\right\}_{h>0}$ is bounded in $L^{4 / 3}\left[0, T ; W^{\prime}\right]$ where the $\iota: H_{0}^{1}(\Omega) \rightarrow W^{\prime}$ is the map $\iota(v)(w)=$ $(v, w)_{L^{2}(\Omega)}$. Lemma 3.2 then shows that $\left\{\iota\left(\bar{v}_{h}\right)\right\}_{h>0}$ is uniformly equicontinuous in $L^{4 / 3}\left[0, T ; W^{\prime}\right]$, and since $\left\{\bar{v}_{h}\right\}_{h>0}$ is bounded in $L^{2}\left[0, T ; H^{1}(\Omega)\right]$, the Lions-Aubin theorem establishes compactness of $\left\{\iota\left(\bar{v}_{h}\right)\right\}_{h>0}$ in $L^{4 / 3}\left[0, T ; W^{\prime}\right]$.

Compactness of $\left\{\bar{v}_{h}\right\}_{h>0}$ in $L^{p}\left[0, T ; L^{2}(\Omega)\right]$ now follows from the following standard argument. Lemma 3.4 shows that for each $\varepsilon>0$ there exists $C(\epsilon)>0$ for which

$$
\left\|\bar{v}_{h}\right\|_{L^{2}(\Omega)} \leq \varepsilon\left\|\bar{v}_{h}\right\|_{H^{1}(\Omega)}+C(\varepsilon)\left\|\iota\left(\bar{v}_{h}\right)\right\|_{W^{\prime}} .
$$

Since $\left\{\bar{v}_{h}\right\}_{h>0}$ is bounded in $L^{2}\left[0, T ; H^{1}(\Omega)\right]$ and $\left\{\iota\left(\bar{v}_{h}\right)\right\}_{h>0}$ is compact in $L^{4 / 3}\left[0, T ; W^{\prime}\right]$, compactness in $L^{4 / 3}\left[0, T ; L^{2}(\Omega)\right]$ follows. Moreover, $\left\{\bar{v}_{h}\right\}_{h>0}$ is bounded in $L^{\infty}\left[0, T ; L^{2}(\Omega)\right]$ so compactness in $L^{p}\left[0, T ; L^{2}(\Omega)\right]$ follows for $1 \leq p<\infty$. 


\subsubsection{Compactness of the director}

Lemma 3.1 and Corollary 3.1 show that the directors $\left\{d_{h}\right\}_{h>0}$ are bounded in $L^{\infty}\left[0, T ; H^{1}(\Omega)\right]$ with derivatives bounded in $L^{2}\left[0, T ; L^{3 / 2}(\Omega)\right]$. The Lions-Aubin lemma then establishes compactness of the directors in $L^{p}\left[0, T ; L^{2}(\Omega)\right], 1 \leq p<\infty$. In order to show that the limit is a solution of the Ericksen-Leslie equations, compactness of $\left\{\bar{d}_{h}\right\}_{h>0}$ in $L^{2}\left[0, T ; H^{1}(\Omega)\right]$ is required. To do this we exploit linearity of the elastic terms in an essential fashion.

Lemma 3.6. Let the hypotheses of Lemma 3.1 hold and assume that the boundary values of the director converge in $H^{1}(\Omega) ; d_{0 h} \rightarrow d_{0}$. Assume that for all $e \in H_{0}^{1}(\Omega)$ there exists $e_{h} \in D_{h}$ such that $e_{h} \rightarrow e$, and that the maximum time step size converges to zero with $h$. Then the directors $\left\{\bar{d}_{h}\right\}_{h>0}$ computed using the discrete weak statement (3.1) are compact in $L^{p}\left[0, T ; H^{1}(\Omega)\right]$ for $1 \leq p<\infty$.

Proof. It suffices to show that it is possible to extract a strongly convergence subsequence. First, pass to a subsequence for which $\bar{d}_{h} \rightarrow \bar{d}$ in $L^{2}\left[0, T ; H^{1}(\Omega)\right]$ and $d_{h} \rightarrow d$ in $L^{2}\left[0, T ; L^{2}(\Omega)\right]$. Since $\bar{d}_{h}$ is a local average of $d_{h}$ it follows that $\bar{d}=d$ and $\bar{d}_{h} \rightarrow d$ in $L^{2}\left[0, T ; L^{2}(\Omega)\right]$. Assumption 3.2 guarantees

$$
w(d, e) \equiv \int_{\Omega}\left(\left[\frac{\partial \mathcal{W}}{\partial \nabla d}\right], \nabla e\right)+\left(\left(\frac{\partial \mathcal{W}}{\partial d}\right), e\right)+(4 / \epsilon)(d, e)
$$

is equivalent to the usual inner product on $H^{1}(\Omega)^{n}$ provided $4 / \epsilon>C_{e}$. Corollary 3.2 then shows that

$$
\int_{0}^{T} w\left(\bar{d}_{h}, \bar{e}_{h}\right)=\int_{0}^{T}\left(G_{h}, \bar{e}_{h}\right)_{L^{2}(\Omega)}, \quad \bar{e}_{h} \in \overline{\mathbb{D}}_{h}
$$

where $\left\{G_{h}\right\}_{h>0}$ is bounded in $L^{2}\left[0, T ; L^{2}(\Omega)\right]$. Passing to a subsequence, we may assume that $G_{h} \rightarrow G$ in $L^{2}\left[0, T ; L^{2}(\Omega)\right]$. The approximation properties of the subspaces $D_{h} \subset H_{0}^{1}(\Omega)^{n}$ allow passage to the limit in the above equation to show

$$
\int_{0}^{T} w(d, e)=\int_{0}^{T}(G, e)_{L^{2}(\Omega)}, \quad e \in L^{2}\left[0, T ; H_{0}^{1}(\Omega)\right] .
$$

Upon recalling that $d_{h}(t)-d_{0 h} \in D_{h}$ it follows that

$$
\begin{aligned}
\int_{0}^{T} w\left(\bar{d}_{h}, \bar{d}_{h}\right) & =\int_{0}^{T} w\left(\bar{d}_{h}, \bar{d}_{h}-d_{0 h}\right)+w\left(\bar{d}_{h}, d_{0 h}\right) \\
& =\int_{0}^{T}\left(G_{h}, \bar{d}_{h}-d_{0 h}\right)_{L^{2}(\Omega)}+w\left(\bar{d}_{h}, d_{0 h}\right) \\
& \rightarrow \int_{0}^{T}\left(G, d-d_{0}\right)_{L^{2}(\Omega)}+w\left(d, d_{0}\right) \\
& =\int_{0}^{T} w\left(d, d-d_{0}\right)+w\left(d, d_{0}\right) \\
& =\int_{0}^{T} w(d, d)
\end{aligned}
$$

Since $\int_{0}^{T} w(d, e)$ is equivalent to the inner product in $L^{2}\left[0, T ; H^{1}(\Omega)\right]$, strong convergence follows, and since $\left\{\bar{d}_{h}\right\}_{h>0}$ is bounded in $L^{\infty}\left[0, T ; H^{1}(\Omega)\right]$ compactness in $L^{p}\left[0, T ; H^{1}(\Omega)\right]$ follows for $1 \leq p<\infty$.

\subsection{Convergence}

The stability and compactness properties of the numerical scheme are sufficient to show that limits of the numerical approximations satisfy the weak statement (2.5)-(2.6) of the Ericksen-Leslie equations. 
Theorem 3.1. Let Assumptions 3.1 hold, and let the elastic energy and dissipative terms satisfy Assumption 3.2. Let $\left\{\left(V_{h}, P_{h}\right)\right\}_{h>0}$ be one of the classical families of div-stable finite element spaces considered in [14], and let $\left\{\mathcal{D}_{h}\right\}_{h>0} \subset H^{1}(\Omega)^{n}$ be a family of classical finite element spaces, and set $D_{h}=\mathcal{D}_{h} \cap H_{0}^{1}(\Omega)^{n}$.

Let the boundary data for the director satisfy $d_{0} \in H^{1}(\Omega)$ and let the initial data satisfy $\left(v^{0}, d^{0}\right) \in L^{2}(\Omega) \times$ $\left(d_{0}+H_{0}^{1}(\Omega)\right)$. Let the data for the momentum equations satisfy $f \in L^{2}\left[0, T ; H^{-1}(\Omega)\right]$ and $m \in L^{2}\left[0, T ; L^{4}(\Omega)\right]$, and assume that the penalty parameter satisfies $C\|m\|_{L^{2}\left[0, T ; L^{4}(\Omega)\right]}^{4} \leq 1 / \epsilon$ where $C$ is the constant depending only upon the viscous and elastic constants guaranteed by Lemma 3.1. Assume that the boundary data $d_{0 h} \in \mathcal{D}_{h}$ for the numerical converges to $d_{0}$ in $H^{1}(\Omega) \cap L^{\infty}(\Omega)$, and that the initial data $\left(v_{h}^{0}, d_{h}^{0}\right)$ converges to $\left(v^{0}, d^{0}\right)$ in $L^{2}(\Omega) \times H^{1}(\Omega)$.

Then solutions $\left(v_{h}, d_{h}\right) \in \mathbb{V}_{h} \times\left(d_{0 h}+\mathbb{D}_{h}\right)$ of the numerical scheme $(3.1)$ are bounded in $L^{\infty}\left[0, T ; L^{2}(\Omega)\right] \times$ $L^{\infty}\left[0, T ; H^{1}(\Omega)\right]$, and weak limits $(v, d) \in L^{2}\left[0, T ; L^{2}(\Omega)\right] \times L^{2}\left[0, T ; H^{1}(\Omega)\right]$ are solutions of the penalized Ericksen-Leslie equations (2.5)-(2.6). In addition, projections $\left(\bar{v}_{h}, \bar{d}_{h}\right) \in \overline{\mathbb{V}}_{h} \times\left(d_{0 h}+\overline{\mathbb{D}}_{h}\right)$ of weakly convergent subsequences converge strongly in $L^{p}\left[0, T ; L^{2}(\Omega)\right] \times L^{p}\left[0, T ; H^{1}(\Omega)\right]$ for $1 \leq p<\infty$.

The proof of this theorem, which we omit, follows upon passing to the limit term-by-term in the discrete weak statement. The technique is standard; the energy estimate establishes the integrability of each term (as in the proof of Lem. 3.5), and strong convergence guaranteed by the compactness of the sequences allows passage to the limit in the nonlinear terms.

\section{REFERENCES}

[1] L. Baňas, A. Prohl and R. Schätzle, Finite element approximations of harmonic map heat flows and wave map into spheres of nonconstant radii. Numer. Math. 115 (2010) 395-432.

[2] J.W. Barrett, X. Feng and A. Prohl, Convergence of a fully discrete finite element method for a degenerate parabolic system modelling nematic liquid crystals with variable degree of orientation. Math. Model. Numer. Anal. 40 (2006) 175-199.

[3] R. Becker, X. Feng and A. Prohl, Finite element approximations of the Ericksen-Leslie model for nematic liquid crystal flow. SIAM J. Numer. Anal. 46 (2008) 1704-1731.

[4] F. Bethuel, H. Brezis and F. Helein, Ginzburg-Landau Vorticies. Kluwer (1995).

[5] R. Cohen, R. Hardt, D. Kinderlehrer, S. Lin and M. Luskin, Minimum energy configurations for liquid crystals: Computational results, in Theory and Applications of Liquid Crystals, J.L. Ericksen and D. Kinderlehrer Eds., The IMA Volumes in Mathematics and its Applications 5, Springer-Verlag, New York (1987).

[6] Q. Du, B. Guo and J. Shen, Fourier spectral approximation to a dissipative system modeling the flow of liquid crystals. SIAM J. Numer. Anal. 39 (2001) 735-762.

[7] F. Duzaar, J. Kristensen and G. Mingione, The existence of regular boundary points for non-linear elliptic systems. J. Reine Angew. Math. 602 (2007) 17-58.

[8] J. Ericksen, Conservation laws for liquid crystals. Trans. Soc. Rheol. 5 (1961) 22-34.

[9] J. Ericksen, Nilpotent energies in liquid crystal theory. Arch. Rational Mech. Anal. 10 (1962) 189-196.

[10] J. Ericksen, Continuum theory of nematic liquid crystals. Res. Mechanica 21 (1987) 381-392.

[11] F.C. Frank, On the theory of liquid crystals. Discuss. Faraday Soc. 25 (1958) 19-28.

[12] G.P. Galdi, An introduction to the mathematical theory of the Navier-Stokes equations I: Linearized steady problems, Springer Tracts in Natural Philosophy 38. Springer-Verlag, New York (1994).

[13] V. Girault and F. Guillén-González, Mixed formulation, approximation and decoupling algorithm for a penalized nematic liquid crystals model. Preprint (2009).

[14] V. Girault, R.H. Nochetto and R. Scott, Maximum-norm stability of the finite element Stokes projection. J. Math. Pures Appl. 84 (2005) 279-330.

[15] R. Hardt and D. Kinderlehrer, Mathematical questions of liquid crystal theory, in Theory and Applications of Liquid Crystals, J.L. Ericksen and D. Kinderlehrer Eds., The IMA Volumes in Mathematics and its Applications 5, Springer-Verlag, New York (1987).

[16] R. Hardt and F.H. Lin, Stability of singularities of minimizing harmonic maps. J. Differential Geom. 29 (1989) 113-123.

[17] R. Hardt, D. Kinderlehrer and F.H. Lin, Existence and partial regularity of static liquid crystal configurations. Comm. Math. Phys. 105 (1986) 547-570.

[18] Q. Hu, X.-C. Tai and R. Winther, A saddle point approach to the computation of harmonic maps. SIAM J. Numer. Anal. 47 (2009) 1500-1523.

[19] R. Jerard and M. Soner, Dynamics of Ginzburg-Landau vortices. Arch. Rational Mech. Anal. 142 (1998) 99-125.

[20] F. Leslie, Some constitutive equations for liquid crystals. Arch. Rational Mech. Anal. 28 (1968) 265-283. 
[21] F. Leslie, Theory of flow phenomenum in liquid crystals, in The Theory of Liquid Crystals 4, W. Brown Ed., Academic Press, New York (1979) 1-81.

[22] F.H. Lin, Mathematics theory of liquid crystals, in Applied Mathematics At The Turn Of Century: Lecture notes of the 1993 summer school, Universidat Complutense de Madrid (1995).

[23] F.H. Lin, Solutions of Ginzburg-Landau equations and critical points of renormalized energy. Ann. Inst. H. Poincaré Anal. Non Linéaire 12 (1995) 599-622.

[24] F.H. Lin, Some dynamic properties of Ginzburg-Landau vorticies. Comm. Pure Appl. Math. 49 (1996) 323-359.

[25] F.H. Lin and C. Liu, Nonparabolic dissipative systems, modeling the flow of liquid crystals. Comm. Pure Appl. Math. XLVIII (1995) 501-537.

[26] F.H. Lin and C. Liu, Existence of solutions for the Ericksen-Leslie system. Arch. Rational Mech. Anal. 154 (2000) $135-156$.

[27] P. Lin, C. Liu and H. Zhang, An energy law preserving $C^{0}$ finite element scheme for simulating the kinematic effects in liquid crystal dynamics. J. Comput. Phys. 227 (2007) 1411-1427.

[28] C. Liu and N.J. Walkington, Approximation of liquid crystal flows. SIAM J. Numer. Anal. 37 (2000) $725-741$.

[29] C. Liu and N.J. Walkington, Mixed Methods for the Approximation of Liquid Crystal Flows. ESAIM: M2AN 36 (2002) 205-222.

[30] G. Mingione, Regularity of minima: an invitation to the dark side of the calculus of variations. Appl. Math. 51 (2006) 355-426.

[31] C.W. Oseen, The theory of liquid crystals. Trans. Faraday Soc. 29 (1933) 883-889.

[32] I.W. Stewart, The Static and Dynamic Continuum Theory of Liquid Crystals: a Mathematical Introduction. Taylor \& Francis Inc., New York (2004).

[33] E.G. Virga, Variational theories for liquid crystals, Appl. Math. Math. Comput. 8. Chapman \& Hall, London (1994).

[34] N.J. Walkington, Compactness properties of the DG and CG time stepping schemes for parabolic equations. SIAM J. Numer. Anal. 47 (2010) 4680-4710. 